\title{
A KML-BASED APPROACH FOR DISTRIBUTED COLLABORATIVE INTERPRETATION OF REMOTE SENSING IMAGES IN THE GEO-BROWSER
}

\author{
Liang Huang ${ }^{\text {a,* }}$, Xinyan Zhu ${ }^{a}$, Wei Guo ${ }^{a}$, Longgang Xiang ${ }^{a}$, Xu Chen ${ }^{\mathrm{b}}$, Yifang Mei ${ }^{\mathrm{a}}$ \\ State Key Laboratory of Information Engineering in Surveying, Mapping and Remote Sensing, \\ Wuhan University, Wuhan 430079, China \\ ${ }^{\mathrm{b}}$ State Key Laboratory of Software Engineering, Wuhan University, Wuhan 430079, China
}

Commission IV, WG IV/5

KEY WORDS: Image Interpretation, Distributed Collaboration, Collaborative interpretation, KML, GeoGlobe

\begin{abstract}
:
Existing implementations of collaborative image interpretation have many limitations for very large satellite imageries, such as inefficient browsing, slow transmission, etc. This article presents a KML-based approach to support distributed, real-time, synchronous collaborative interpretation for remote sensing images in the geo-browser. As an OGC standard, KML (Keyhole Markup Language) has the advantage of organizing various types of geospatial data (including image, annotation, geometry, etc.) in the geo-browser. Existing KML elements can be used to describe simple interpretation results indicated by vector symbols. To enlarge its application, this article expands KML elements to describe some complex image processing operations, including band combination, grey transformation, geometric correction, etc. Improved KML is employed to describe and share interpretation operations and results among interpreters. Further, this article develops some collaboration related services that are collaboration launch service, perceiving service and communication service. The launch service creates a collaborative interpretation task and provides a unified interface for all participants. The perceiving service supports interpreters to share collaboration awareness. Communication service provides interpreters with written words communication. Finally, the GeoGlobe geo-browser (an extensible and flexible geospatial platform developed in LIESMARS) is selected to perform experiments of collaborative image interpretation. The geo-browser, which manage and visualize massive geospatial information, can provide distributed users with quick browsing and transmission. Meanwhile in the geo-browser, GIS data (for example DEM, DTM, thematic map and etc.) can be integrated to assist in improving accuracy of interpretation. Results show that the proposed method is available to support distributed collaborative interpretation of remote sensing image.
\end{abstract}

\section{INTRODUCTION}

As the development of sensor and earth observation technologies, earth observation system has taken shape and perfected gradually. Multi-scale and multi-spectral aerospace remote sensing platforms carry out consistent observations of earth. These platforms have continually producing multitemporal observational data. Tremendous data enrichment, calls for efficient information extraction technologies to discover underlying useful knowledge. Quantitative analysis and image interpretation are two effective methods of extracting information from remote sensing images (Richards and Jia, 2006). Image interpretation is a process of extracting target information by analysing, comparing, reasoning various image features comprehensively, based on imaging theories and professional knowledge (e.g. Geology, Surveying and Mapping). Image interpretation has experienced four stages of development that are visual interpretation, interactive interpretation, knowledge based expert interpretation and automatic interpretation. During this process, image interpretation methods have been more automatic and intelligent. Practices of interpretation, however, have been stand-alone operations. Multiple workers finish common interpretation task in a serial way. As the improving of accuracy and size of earth observation data, image interpretation task becomes increasingly complex and difficult. Traditional mode of serial working cannot satisfy the demands of efficiency, while large-scale interpretation task goes. Since interpreters lack of effective communication and resources sharing, the accuracy of image interpretation is deeply influenced by personal experience and professional knowledge background. Inconsistencies exist among interpretation results without communication. Thus the transformation of interpretation practices is necessary. Collaborative interpretation is an efficient way to improve efficiency and accuracy by cooperative work. Multiple interpreters work synchronously or asynchronously in a distributed environment can reduce working time. Fully sharing of ideas and resources helps to improve the accuracy of interpretation results. Computer Supported Cooperative Work (CSCW) is one of the most common technologies to implement cooperative work. With $\mathrm{CSCW}$, geographically dispersed researchers can complete a task cooperatively. In the area of medicine, some scholars introduce $\mathrm{CSCW}$ to support collaborative interpretation of different medical image data for teleconsultation and analysis (Andersen et al., 2011; Hu et al., 2007; Zhang et al., 2000; Zhang et al., 2004). (Carlos et al., 2000) integrates CSCW and image segmentation module to design a collaborative environment called diSNet for volume data analysis. (Lim et al., 2001) combines Java applets and CSCW to provide a webbased collaborative system for medical image analysis and diagnosis. (Huang et al., 2009) uses web services approach to build a medical image synchronous collaborative analysis system, which enables distributed physicians doing

*Corresponding author. Tel: +1 347707 0410;

Email Address: plaquemine@whu.edu.cn (L. Huang) 
collaborative analysis on medical image. Considering successful applications of CSCW in the area of medicine, it makes sense to introduce CSCW for collaborative interpretation of geospatial data (e.g. remote sensing images). (Baraghimian and Young, 2001) proposed a virtual collaborative software environment called GeoSpace ${ }^{\mathrm{TM}}$ by combining improved InfoWorkSpace ${ }^{\mathrm{TM}}$ and GIS analysis functionalities. It mainly provides interactive analysis and visualization of geospatial information for collaborators. (Liu, 2004) develops a group-based image interpretation system for remote sensing applications. In this system, Windows Netmeeting tool is employed to implement information communication and application sharing among interpreters. (Convertino et al., 2005) integrates CSCW and Multiple View Visualizations to present a collaborative visualization framework for distributed and synchronous teamwork. (Xu, 2005) develop Socket messages of fixed format to pass cooperative perception among interpreters. (Austerschulte and Keßler, 2010) integrates several tools (ArcPad, GPS, etc.) to build a remote collaborative system that supports information sharing among the teams participating in geological data gathering. (Di Ciaccio et al., 2011) adopts webservices and other open standards and libraries to provide collaboration environment for disaster incident management. (Xu et al., 2011) gives redefinition and conceptual framework of collaborative virtual geographic environment to support geocollaboration. All researches above are related work in collaborative image interpretation. Different technologies have been adopted to implement collaboration. However some shortcomings exist. For example, web service needs plenty of network resource and the server bottleneck exists. Specific Socket messages are hard to be extended. Windows Netmeeting tool is inefficient to share application in the WAN. Besides, all existed systems are hard to manage and visualize massive largesize images and referential information. In this paper, a KMLbased approach is proposed for distributed collaborative interpretation of remote sensing images in the geo-browser. KML, as an OGC standard, is an xml-based file format used to display geographic data in a geo-browser. It supplies advances in extensibility and less data quantity. This article employs KML to share interpretation results and operations among interpreters. Meanwhile the geo-browser such as Google Earth has advantages in effective management, analysis and visualization of massive spatial data. It is appropriate to provide collaborators with unify interface and analysis tools of image browsing, operation and interpretation.

\section{DESIGN OVERVIEW}

\subsection{Architecture of Collaborative Image Interpretation}

Firstly, this article will present the architecture of collaborative image interpretation. In order to provide collaborative interpretation environment in the geo-browser, some constraints are required. Firstly, data transmitting of large-scale image among collaboration clients should be avoided. It promises no limitation of network bandwidth. Secondly, it is prefer to conduct image processing in server-side. Moreover, geographically distributed interpreters can participate collaboration interpretation anytime and anywhere with a geobrowser. Finally, all interpreters can interactively communicate and share interpretation results with each other. In this paper, the architecture of collaborative image interpretation is composed of three tiers: collaboration client tier, spatial data server tier and collaboration sever tier (Figure 1). Collaboration client tier contains four main modules. There are spatial data visualization module, vector interpretation module, communication module and collaboration module. Spatial data visualization module is designed to display image, vector, annotation, DEM (digital elevation model) and other spatial data. Task images and results of vector interpretation are visualized in this module. Vector interpretation module provides various vector tools (point icon, annotation, polyline, polygon and compound geometry) to indicate targets in the image. Communication module is responsible for Socket messages communication among interpreters. All Socket messages are delivered to specified clients via collaboration server. Collaboration module implements cooperative work and collaboration awareness. Spatial data server tier is composed of one catalog server and several geospatial data servers. Geospatial data server provides tiles service of various spatial data, such as image, vector, DEM, annotation, etc. All geospatial data servers register published tile services in the catalog server. Catalog server responses to spatial data requests and invokes registered tile services to obtain spatial data for clients. Collaboration server mainly implements three collaboration related services that are collaboration launch service, collaboration perceiving service and communication service. Collaboration launch service enables any collaboration client to create a collaborative image interpretation task. Task images and cooperative interpreters' information are uploaded from the client. Collaboration perceiving service is called to share clients' interpretation operations and results in the form of KML files. Communication service delivers Socket messages among collaboration interpreters.

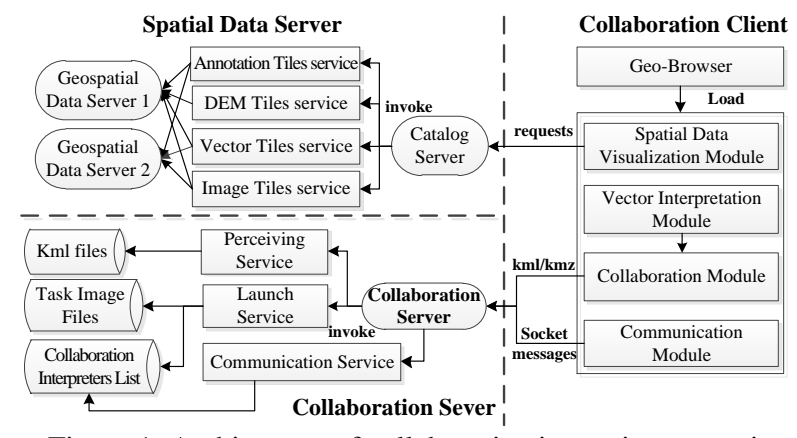

Figure 1. Architecture of collaborative image interpretation

\subsection{Collaboration Mode}

In this section, the work mode of collaborative image interpretation will be detailed. Different from existed methods (Liu, 2004; Xu, 2005), this article adopts KML-based collaboration mode (Figure 2). In KML-based collaboration mode, any client could launch a collaboration interpretation task. The sponsor firstly selects remote sensing images and appropriate online interpreters. All these initial information are uploaded into collaboration server via collaboration launch service. Collaboration server responses to the service and performs image pre-processing, such as projection transformation, invalid pixel value elimination, image pyramid creation, etc. After that, collaboration server delivers task description and image configuration to specific clients. A uniform interpretation interface will be prepared for collaboration clients. Each client employs provided vector tools to accomplish image interpretation according to professional knowledge and personal experience. When ambiguity exists, interpreters could communicate each other with Socket messages (Maybe third-party voice software will provide better communication). Meanwhile collaboration awareness service 
helps to share interpretation results in the form of KML files among them. When interpretation finished, results are committed to the sponsor client for integration and conflict check. If conflict exists, all participants discuss via messages and vote for final results. Final results are described by KML elements and cached as KML files. Compared to existed method, KML-based approach has the following advantages: 1) Remote sensing images and interpretation results are stored separately. Interpretation will not destroy original images. 2) Interpretation results in the form of KML are convenient to share and interoperate among geo-browsers. 3) Using KML elements to describe interpretation results occupies less storage space than using picture.

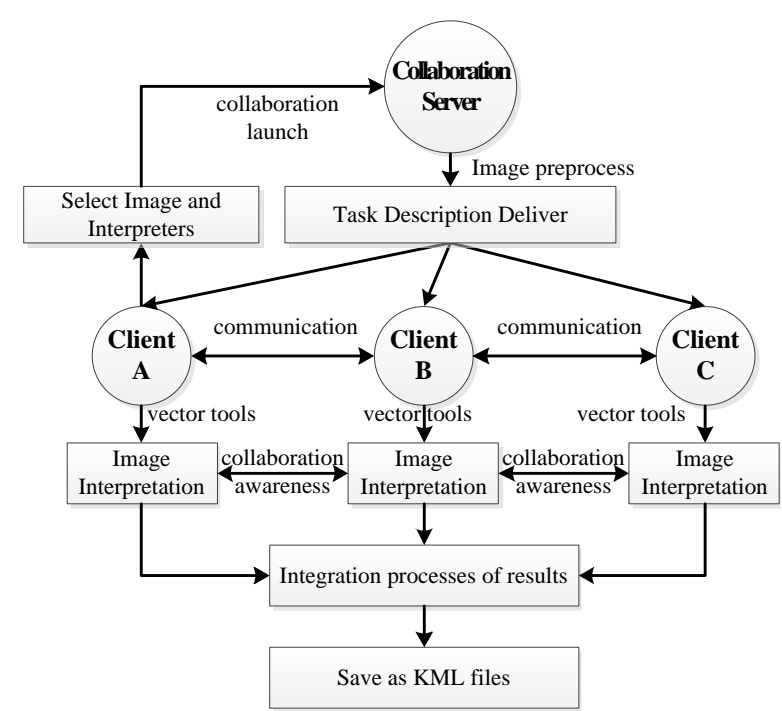

Figure 2. Collaboration mode

\section{METHOD}

\subsection{KML-based Collaborative Interpretation Description}

In this paper, KML elements are mainly employed to describe collaboration interpretation launch and awareness information. Collaboration interpretation launch information contains remote sensing images and interpreters. Collaboration awareness information is composed of geometries, overlays, interpreters and operations. Published KML elements can only be used to describe geometries and overlays. This article expands KML to describe image metadata, image operations and interpreter information. Figure 3 is an overview of main KML elements adopted for interpretation. In the diagram, elements shown in boxes are elements not actually used in KML files. They are a useful way for a single element to serve as the programmatic foundation for multiple similar derived elements. In the published KML elements, <Feature> element organizes $<$ Placemark> and <Overlay> elements to describe display style of an object in geo-browser. <Placemark> element contains at least one of styles in <Geometry> elements to describe vector objects. <Overlay> element describes raster objects on 3D virtual earth surface or $2 \mathrm{D}$ screen. <TimePrimitive> element is used to express a single moment or extent in time. $<$ LatLonBox> element depicts space range of object in geodetic coordinate system. This article adds some new elements. $<$ CoInterpretation> element indicates a collaboration interpretation task is launched. <CoAwareness> element describes vector interpretation results of a client. 〈rsImage $\rangle$ and <interpreter> elements respectively indicates images and clients (in the form of IP address) for interpretation. <BandCombination>, <GreyTransformation> and $<$ GeometricCorrection $>$ are elements used for image process.

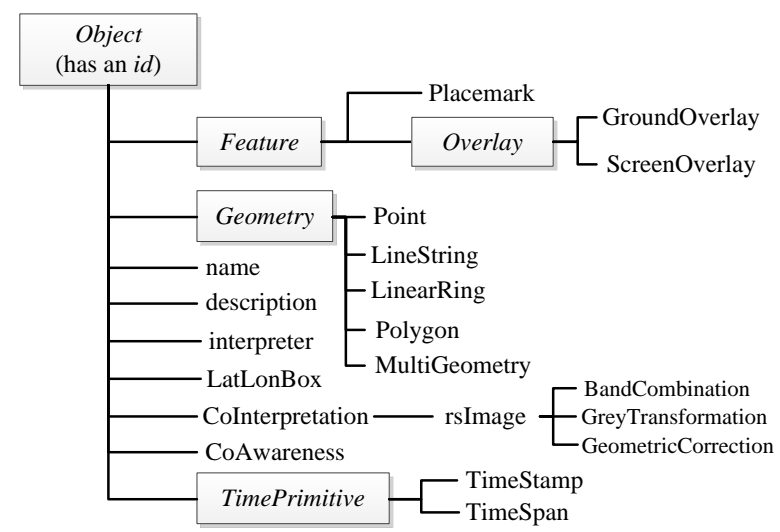

Figure 3. Main KML elements adopted for interpretation

Next this article will introduce how to use elements above to describe collaboration launch and awareness information. For collaboration interpretation launch description, a <name> element is needed to describe subject. More than one <rsImage> elements are used to present images for interpretation. A group of <interpreter> elements indicates clients to complete task. It is optional to adopt a <description> element to introduce image metadata, such as data source, reference coordinate system, spatial resolution, spectra, etc. Figure 4 is an instance of collaboration interpretation launch described in KML.

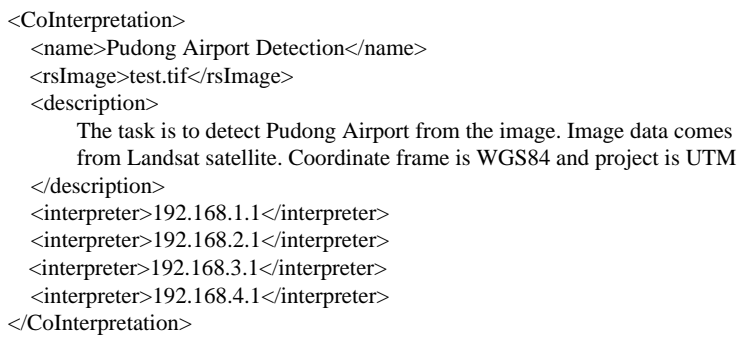

Figure 4. KML description of Collaboration launch

For collaboration awareness description, <interpreter> and $<$ TimeStamp> elements are needed to tell where and when the interpretation results are captured. A group of <Placemark $>$ elements are employed to describe interpretation results. Each placemark can be either geometry or overlay. A <rsImage> element gives an image has been interpreted. Moreover, some image processing operations are optional. For example, original image sometimes needs geometric correction to obtain precise object coordinate. In this case, <GeometricCorrection> element can be used like the "Row, Column, Lat, Lon". "Row" and "Column" represent pixel position of a point in an image. "Lat" and "Lon" represent geospatial coordinates of the point. Figure 5 is an instance of collaboration awareness description in KML. 


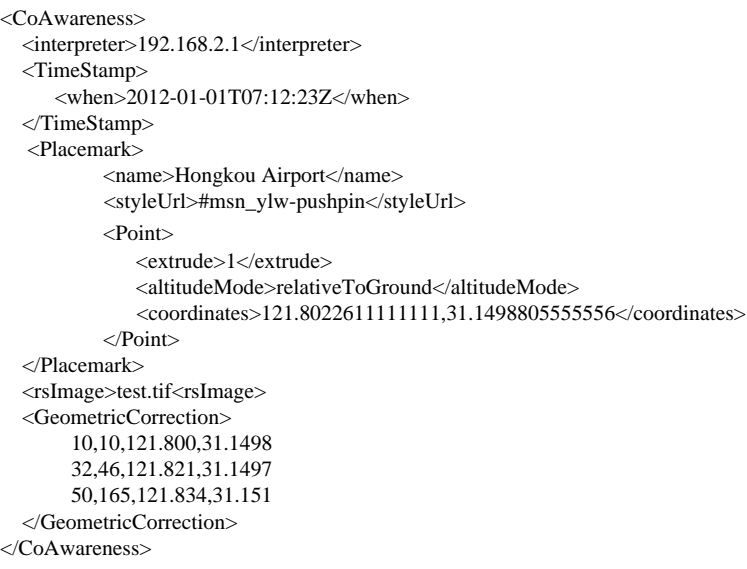

Figure 5. KML description of Collaboration awareness

\subsection{Collaboration Related Service}

In order to support collaboration interpretation, three collaboration related services were developed. They are launch service, perceiving service and communication service. All services are deployed on collaboration server. Launch service supports to create an image collaboration interpretation. After selecting images and interpreters, user invokes launch service to upload the information in the form of $\mathrm{KML} / \mathrm{KMZ}$ file. Collaboration sever create a collaboration folder in response to launch service. A collaboration folder includes one task subfolder and several interpreter subfolders. In the task subfolder, KML/KMZ files describing task images and interpreters information are stored. In addition, task images go through some processing operations, such as projection transformation, background elimination, and image pyramid generation. The interpreter subfolders store $\mathrm{KML} / \mathrm{KMZ}$ files describing interpretation results for interpreters respectively. After collaboration folder was created, collaboration server prepares task images for each interpreter. All clients have a unified interpretation interface. Perceiving service supports to share interpretation results in KML files among interpreters. An interpreter can either share his own interpretation results or obtain others'. If sharing results, an interpreter invokes perceiving service to upload KML files. Collaboration server stores the KML files to some or all interpreter subfolders and configures the results to clients for visualization. If obtaining results, an interpreter invokes perceiving service to request KML files from specified interpreters. Collaboration server requests for interpretation results by sends messages to specified interpreters and transmits results to the requestor. Communication service supports to provide written communication among interpreters using Socket messages. On the interpretation interface, there is an interpreters list. An interpreter can select one or more co-operators for communication in a chat window. All messages are conveyed to interpreters via collaboration server.

\section{EXPERIMENTS AND DISCUSSION}

In order to show the availability of the proposed method, this article performs some experiments of collaborative image interpretation on GeoGlobe. The GeoGlobe is an extensible and flexible geospatial platform for managing and visualizing massive geospatial information. It can provide distributed users with quick browsing and transmission of geospatial data. Here this article shows an example of collaboratively identify
LIESMARS (State Key Laboratory of Information Engineering in Surveying, Mapping and Remote Sensing) of China from a multi-spectral image. Three collaboration clients (called A, B and $\mathrm{C}$ ) are employed to complete interpretation task. Client A selected task image and launched the collaboration interpretation. Client B identified the position of LIESMARS using cyan star icon and annotation. The annotation states interpretation result and corresponding interpreter. Moreover a detailed introduction of LIESMARS will be given by clicking the icon. Client $\mathrm{C}$ drew the coverage of LIESMARS using yellow polygon and annotation. Results of both client $\mathrm{B}$ and $\mathrm{C}$ are updated to Client A. As a sponsor, client A discusses with other clients and votes for final results. Then final results are saved as KML/KMZ files. Figure 6 is a screenshot of interpretation results.

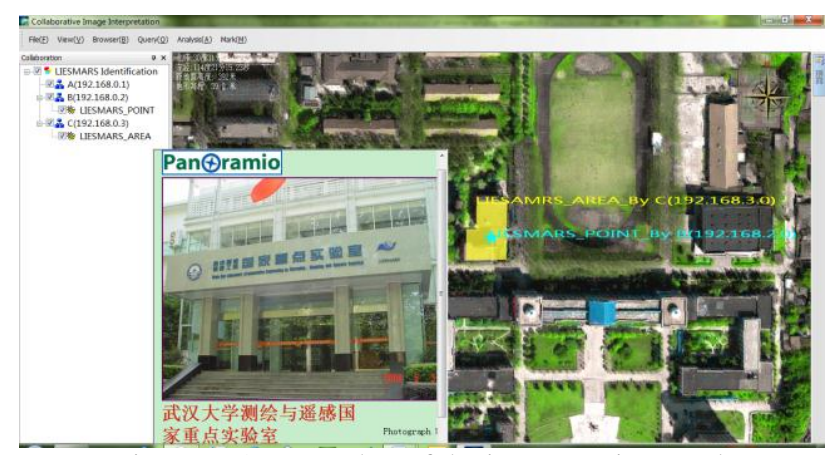

Figure 6. A screenshot of the interpretation result

It illustrates that KML is available for collaborative image interpretation. In addition, this method alleviates some drawbacks of existed methods for collaborative image interpretation. For image transfer, existed methods need transfer task image file to all clients, which results in a high burden to the network. KML-based method transfers image tiles instead of image file. An image file may be more than a few MB or GB, and an image tile may be a few KB. An image file only divides into several tiles. Therefore, KML-based method brings a low burden of the network. For collaboration awareness, existed methods use either specified messages or application sharing. Specified messages are hard for expansion. And application sharing spends on lots of computing and network resource. However, KML is convenient to be expanded for personalized application. For example, users can define new geometry element to describe complex plotting symbols. It provides better performance on collaboration awareness. Moreover, existed method saves interpretation results in image. If sharing results, it needs to transfer image file. But for the proposed method, it only shares KML files without image. Through the comparison, KML-based method applied in collaborative image interpretation has greater advantages than existed methods.

\section{CONCLUSION}

Existed methods for collaborative interpretation of remote sensing images have many drawbacks, such as burden network, inefficient sharing of interpretation results, slow browsing. This article proposed a KML-based approach to improve these problems. Experience results illustrates that KML-based method provides better performance for collaborative image interpretation. The KML has an advantage in describing various spatial data. Also as an OGC standard, it is convenience to be expanded and shared. It is possible that using KML to describe and share interpretation results. Meanwhile, geo-browser can 
provide quick browsing of spatial data described in KML. In a geo-browser, all data are transmitted in the form of tile, which reduces the burden of network. This study represents just the first step in implementing collaborative interpretation of remote sensing image. As such, it has many limitations. For example, vector symbols for interpretation are too simple and few. An efficient conflict resolution mechanism is lacked. In future work, it is a challenge to design a symbol library for image interpretation, including providing KML elements descriptions and symbol tools.

\section{ACKNOWLEDGEMENTS}

This research was supported by "National Natural Science Foundation of China Grant 40971232 and 41023001"; "National Key Technology R\&D Program of China Grant 2012BAH35B03"; "National High Technology Research and Development Program of China Grant 2011AA010500"; "Fundamental Research Funds for the Central Universities of China 20106190201000148".

\section{REFERENCES}

Andersen, T., Bjørn, P., Kensing, F. and Moll, J., 2011. Designing for collaborative interpretation in telemonitoring: Reintroducing patients as diagnostic agents. International Journal of Medical Informatics, 80(8): e112-e126.

Austerschulte, M. and Keßler, C., 2010. Suggestive GeoTagging Assistance for Geo-Collaboration Tools., 0: 143-162.

Baraghimian, T. and Young, M., 2001. GeoSpaces - A virtual collaborative software environment for interactive analysis and visualization of geospatial information, 2001 International Geoscience and Remote Sensing Symposium (IGARSS 2001). International Geoscience and Remote Sensing Symposium (IGARSS). Institute of Electrical and Electronics Engineers Inc., Sydney, NSW, Australia, pp. 1678-1680.

Carlos, A. et al., 2000. disnei: A collaborative environment for medical images analysis and visualization.

Convertino, G., Ganoe, C.H., Schafer, W.A., Yost, B. and Carroll, J.M., 2005. A multiple view approach to support common ground in distributed and synchronous geocollaboration, Coordinated and Multiple Views in Exploratory Visualization, 2005. (CMV 2005). Proceedings. Third International Conference on, pp. 121- 132.

Di Ciaccio, R., Pullen, J. and Breimyer, P., 2011. Enabling distributed command and control with standards-based geospatial collaboration, 11th IEEE International Conference on Technologies for Homeland Security, HST 2011, November 15, 2011 - November 17, 2011. 2011 IEEE International Conference on Technologies for Homeland Security, HST 2011. IEEE Computer Society, Waltham, MA, United states, pp. 512517.

Hu, Y., Haynor, D.R. and Maravilla, K.R., 2007. WHIPPET - : a collaborative software environment for medical image processing and analysis., $8(31)$.

Huang, Y., ChunYang, H., Yongwang, Z. and Dianfu, M., 2009. Web-based remote collaboration over medical image using web services, Information Infrastructure Symposium, 2009. GIIS '09. Global, pp. 1-8.

http://code.google.com/intl/zh-

$\mathrm{CN} / \mathrm{apis} / \mathrm{kml} /$ documentation/kmlreference.html (2010)

Lim, Y.S., Feng, D.D. and Cai, T.W., 2001. A web-based collaborative system for medical image analysis and diagnosis, Selected papers from the Pan-Sydney workshop on Visualisation - Volume 2. Australian Computer Society, Inc., Sydney, Australia, pp. 93-95.

Liu, Y., 2004. Study on Grouping Interpretation Technology for Remote Sensing Images, Institute of Remote Sensing Applications Chinese Academy of Sciences, Beijing.

Richards, J.A. and Jia, X., 2006. Remote Sensing Digital Image Analysis: An Introduction 4th Edition, 46. Springer, New York, $439 \mathrm{pp}$.

$\mathrm{Xu}$, B. et al., 2011. Collaborative virtual geographic environments: A case study of air pollution simulation. Information Sciences, 181(11): 2231-2246.

$\mathrm{Xu}, \mathrm{G} ., 2$ 2005. Research and Implementation of Cooperative Remote Sensing Image Interpreting System, Institute of Electronics Chinese Academy of Sciences, Beijing.

Zhang, J. et al., 2000. Real-time teleconsultation with highresolution and large-volume medical images for collaborative healthcare. Information Technology in Biomedicine, IEEE Transactions on, 4(2): 178-185.

Zhang, R. et al., 2004. A telemedicine system for collaborative work on radiographic coronary video-images. Journal of Telemedicine and Telecare, 10(3): 152 -155. 